\title{
SERS-active Au@Ag core-shell nanorod (Au@AgNR) tags for ultrasensitive bacteria detection and antibiotic-susceptibility testing
}

\author{
Liyan $\mathrm{Bi}^{\mathrm{a}}{ }^{, *}, 1$, Xiao Wang ${ }^{\mathrm{a}, 1}$, Xiaowei Cao ${ }^{\mathrm{b}}$, Luying Liu ${ }^{\mathrm{a}}$, Congcong Bai ${ }^{\mathrm{a}}$, Qingyin Zheng ${ }^{\mathrm{a}}$, \\ Jaebum Choo ${ }^{\mathrm{d}, * *}$, Lingxin Chen ${ }^{\mathrm{a}, \mathrm{c}, \mathrm{e},{ }^{* * *}}$ \\ a Transformative Otology and Neuroscience Center, Binzhou Medical University, Yantai 264003, China \\ ${ }^{\mathrm{b}}$ Institute of Translational Medicine, Medical College, Yangzhou University, Yangzhou 225001, China \\ ${ }^{c}$ CAS Key Laboratory of Coastal Environmental Processes and Ecological Remediation, Shandong Key Laboratory of Coastal Environmental Processes, Yantai Institute of \\ Coastal Zone Research, Chinese Academy of Sciences, Yantai 264003, China \\ d Department of Chemistry, Chung-Ang University, Seoul 06974, South Korea \\ ${ }^{\mathrm{e}}$ School of Pharmacy, Binzhou Medical University, Yantai 264003, China
}

\section{A R T I C L E I N F O}

\section{Keywords:}

$\mathrm{Au} @ \mathrm{AgNR}$

SERS nanotag

Antibiotic susceptibility testing

Minimum inhibitory concentration

Principal component analysis

\begin{abstract}
A B S T R A C T
There is a challenge to obtain an ultrasensitive and rapid approach to detect bacteria and identify resistance. As a powerful bioanalytical tool, surface-enhanced Raman scattering (SERS) in bacterial detection have attracted increasing attentions. Herein, we developed a SERS-active Au@Ag core-shell nanorod (Au@AgNR) tag platform for ultrasensitive bacteria detection and antibiotic-susceptibility testing (AST). The platform established that surface enhanced Raman scattered Rhodamine 6G (R6G) absorption at $1517 \mathrm{~cm}^{-1}$ had a good linearity (RI = $3865+193 \log C ; \mathrm{R}^{2}=0.97$ ) with logarithm of $E$. coli concentration over a range of $10^{7}-10^{2} \mathrm{CFU}$ (colony forming unit) $/ \mathrm{mL}$ with limit of detection as low $10^{2} \mathrm{CFU} / \mathrm{mL}$. When $E$. coli was exposed to ampicillin at minimum inhibitory concentration (MIC, $4 \mu \mathrm{g} / \mathrm{mL}$ ), Raman spectroscopy showed the obvious variation between ampicillinsusceptible E. coli (Amp ${ }^{-}$-E. coli) and the ampicillin-resistant E. coli (Amp ${ }^{+}-E$. coli). Combined with principal component analysis (PCA) statistical analysis, the Raman intensity variation mentioned above allows to obtain rapid antibiotic resistance testing $(<3.5 \mathrm{~h})$. In addition, E.coli spiked into blood from C57BL/6 mice can be identified clearly, indicating the potential for point-of-care diagnostics.
\end{abstract}

\section{Introduction}

Infection by pathogenic bacteria is threatening public health [1], especially for antibiotic-resistant bacteria, which has always been a global healthcare issue [2]. Therefore, a sensitive and accurate approach that can speed up the pathogens detecting and antibiotic susceptibility testing is highly desirable in clinical applications. That will be benefit for halting the spread of pathogen related diseases at source [3] and avoiding abuse of antibiotic [4,5]. The conventional assays for bacteria detection relies on plating and culturing, which takes days to confirm the results and lack specificity [6]. Due to the intrinsic rich optical properties and chemical stability of nanocrystal [7], over past years, some biosensor based on nanocrystals for bacteria detection have been widely proposed, such as fluorescence [8], light-scattering [9], colorimetric [10], surface-enhanced Raman scattering (SERS) [11,12] and so on. Among these methods, SERS as a readout method has been proven as the most promising tool, which is unique in high detection sensitivity, nondestructive data acquisition and intrinsic spectroscopic fingerprints. SERS-based biosensor is not only exploited for analyzing pathogen bacteria but also for antibiotic susceptibility testing [13]. In the past couples of years, researchers have proposed two types of SERS-based bioanalysis, which are label-free detection $[14,15]$ and label-based detection, respectively $[16,17]$. The label-free detection is based on their intrinsic vibrational information of pathogen bacteria. The label-based detection is also commonly used to detect bacteria, which utilizes Raman label molecules as reporter for discriminating pathogen.

\footnotetext{
* Corresponding author.

$* *$ Corresponding author.

$* * *$ Corresponding author.

E-mail addresses: liyan_bi@163.com (L. Bi), jbchoo@cau.ac.kr (J. Choo), lxchen@yic.ac.cn (L. Chen).

1 These authors contributed equally to this work.
} 
In the two kinds of SERS-based biosensors, the pathogen is adsorbed in the vicinity of nanocrystals, or on the surface of noble metal [18]. Compared with label-free detection, label-based detection is more sensitive and complex, which is comprised of SERS-encoded nanoparticles and chemoselective receptors. Chen et al. fabricated multifunctional Au@Rubpy/GO SERS tags for targeting E.coli via electrostatic adsorption [19]. Alvarez-Puebla et al. demonstrated SERS based biosensor for detection of $S$. aureus using aptamers and antibodies to recognize bacteria [20]. Faulds et al. illustrated assay format including magnetic nanoparticles and SERS nanotag, employing antibodies for recognition of bacteria [21]. Among these approaches, the main focus is to improve the capture efficiency. That is to say, nanocrystals are capable of anchoring onto the bacteria surface as much as possible [22]. In this regard, strong noncovalent interaction between streptavidin and biotin is more attractive. The specific immunochemistry can occur between streptavidin-functionalized nanoparticles and biotinylated antibody, which is used to enhance orientation and attachment during targeting onto the bacteria [23]. Herein, we developed SERS-active Au@Ag core-shell nanorod (Au@AgNR) tag systems for bacteria detection and antibiotic susceptibility testing, which is based on streptavidine-biotin system [24]. Au@AgNRs are functionalized with Raman label and streptavidine, forming the Au@AgNR@SA complex. The biotinylated antibodies can be specially linked to the Au@AgNR@SA complex via strong noncovalent interaction, forming the complete SERS nanotags (Au@AgNR@SA@Ab), which can specifically anchor onto E.coli. This platform provides highly-accessible hotspots to enhance SERS and enriches bacteria efficiently. In our work, E. coli are selected as the model bacteria. Combined with PCA statistical analysis, the SERS nanotag systems can allow to obtain rapid antibiotic resistance testing. Furthermore, E.coli spiked into blood from C57BL/6 mice can be identified clearly, indicating the potential for clinical routine.

\section{Experimental section}

\subsection{Materials and reagents}

Hydrogen tetrachloroauric acid $\left(\mathrm{HAuCl}_{4} \cdot 3 \mathrm{H}_{2} \mathrm{O}, 99.9 \%\right)$, hexadecyltrimethylammonium bromide (CTAB, 99\%), sodium borohydride $\left(\mathrm{NaBH}_{4}\right)$, L-ascorbic acid (AA), and silver nitrate salt $\left(\mathrm{AgNO}_{3}, 99.97 \%\right)$, Luria-Bertani (LB), absolute ethanol were purchased from Sinopharm Chemical Reagent Co., Ltd (Shanghai, China). Hydrochloric acid ( $\mathrm{HCl}$ ) was purchased from Zhongshi Chemical Co., Ltd (Shanghai, China). Hexadecyltrimethylammonium chloride (CTAC,97\%), rhodamine 6G (R6G), DL-thioctic acid (LA), $N$-hydroxysuccinimide (NHS), glutaraldehyde (50\%) were all purchased from Aladdin Bio-Chem Technology Co., Ltd (Shanghai, China). Streptavidin were purchased from Solarbio technology Co., Ltd. (Beijing, China). Biotinylated antibody was purchased from Bioss Biotechnology co., Ltd (Beijing, China). $\mathrm{N}_{2}$ was purchased from Yantai Rulin Gas Co., Ltd (Yantai, China). Ampicillin sodium salt was purchased from Shanghai Macklin Biochemical Co.,Ltd (Shanghai, China). E.coli DH5 $\alpha$ was purchased from Takara Biomedical Technology Co., Ltd (Beijing, China). The reagents mentioned above were used as received without any treatment and performed with MilliQ water.

\subsection{E.coli culturing}

In this work, bacterial strains utilized included E.coli DH5 $\alpha$ strain (Amp ${ }^{-}$- E.coli) and E.coli DH5 $\alpha$ strains with ampicillin resistance profile $\left(\mathrm{Amp}^{+}-\right.$E.coli). The Amp ${ }^{+}$- E.coli grows from E. coli DH5 $\alpha$ and displays ampicillin-resistant phenotype [25]. The bacterial strains were cultured in LB medium at $37^{\circ} \mathrm{C}$ with continuous shaking at $200 \mathrm{rmp} / \mathrm{min}$ for $16 \mathrm{~h}$ [26]. Afterward, $1 \mathrm{~mL}$ bacteria were collected via centrifugation at 6000 rpm for $5 \mathrm{~min}$ in room temperature. The $E$. coli solution was cleaned twice and eventually resuspended into PBS buffer. $1 \mathrm{~mL}$ of $2.5 \%$ glutaraldehyde was injected into bacteria for $30 \mathrm{~min}$. Prior to analysis, bacteria concentration was determined via optical density (OD) at 600 $\mathrm{nm}\left(\mathrm{OD}_{600}\right)$.

\subsection{The Experiment's animals}

$0.2 \mathrm{~mL}$ of blood sample was collected from inner canthus of C57BL/6 mice. As experimental animal C57BL/6 mice (female, 5-7 weeks, 18-20g) were kept at the animal house at Binzhou Medical University, which is standard condition [27]. All of the procedures involving C57BL/ 6 mice were approved by the Institutional Animal Use and Care Committee of Binzhou Medical University.

\subsection{Synthesis of Au@Ag core-shell nanorods (Au@AgNR)}

Au nanorods were fabricated according to seed-mediated method previously reported [27]. The concentration of colloidal suspensions was estimated to be $1.50 \mathrm{nM}$ [28]. The Au@AgNRs were fabricated via modified Wang's report [7]. The procedure is as follows: $30 \mathrm{~mL}$ of $\mathrm{Au}$ nanorod solution was enriched with centrifuging at $8000 \mathrm{rpm}$ for $15 \mathrm{~min}$ and then was dispersed into $30 \mathrm{~mL} 0.08 \mathrm{M}$ CTAC. Afterward, $18 \mathrm{~mL} 0.01$ $\mathrm{M} \mathrm{AgNO}_{3}$ and $9 \mathrm{~mL} 0.1 \mathrm{M}$ ascorbic acid were added into the solution mentioned above, successively. The resultant solution was placed in water bath at $65^{\circ} \mathrm{C}$ for $4 \mathrm{~h}$. After addition of the ascorbic acid, the color changes from pink into russet within 20 min, illustrating the silver nanoparticle is rapidly coating onto Au nanorods.

\subsection{SERS platform for bacteria detection}

Fig. 1 showed the protocol for designing SERS nanotags as follows: $57 \mathrm{~mL}$ Au@AgNR solution was incubated with $5 \mathrm{~mL}$ of $10^{-3} \mathrm{M} \mathrm{R6G}$ and $5 \mathrm{~mL}$ of $4 \mathrm{mg} / \mathrm{mL}$ LA in $\mathrm{N}_{2}$ condition overnight [29]. To activate carboxylate groups in LA, the suspension was treated with $0.05 \mathrm{M}$ NHS and $0.2 \mathrm{M} \mathrm{EDC}$ for $2 \mathrm{~h}[30,31]$. The activated Au@AgNR was purified via centrifuging at $7000 \mathrm{rpm}$ for $15 \mathrm{~min} .10 \mu \mathrm{l}$ of $1 \mathrm{mg} / \mathrm{mL}$ streptavidine was added into $1 \mathrm{~mL}$ Au@AgNR solution mentioned above for $1 \mathrm{~h}$, forming streptavidine-functionalized Au@AgNRs (Au@AgNR@SA). And then 1 $\mu \mathrm{l}$ of $0.1 \mathrm{mg} / \mathrm{mL}$ biotinylated antibody was incubated with the Au@AgNR@SA for $1 \mathrm{~h}$, forming the sandwich nanohybrids (Au@AgNR@SA@Ab) [32].1 mL of Au@AgNR@SA@Ab colloids was spiked into the $1 \mathrm{~mL}$. coli solution ranging from $10^{2}$ to $10^{7} \mathrm{cfu} / \mathrm{mL}$ and incubated at $37^{\circ} \mathrm{C}$ for $1 \mathrm{~h}$ with shaking at $200 \mathrm{rpm} / \mathrm{min}$ and $37^{\circ} \mathrm{C}($ E.coli complex). Free bioconjungates were removed by centrifugation.

\subsection{Spectral preprocessing and data analysis}

After incubation, Raman spectra were collected from glass capillary, which absorbed E.coli complex at different concentration mentioned above. The laser beam was focused on the glass capillary. As shown in Fig. 1C, 5 random spots on glass capillary were scanned for repeat SERS measurement. The SERS nanotag was used for detecting the bacteria in mouse blood, in which the concentration was down to $10^{4} \mathrm{CFU} / \mathrm{mL} .10^{4}$ $\mathrm{CFU} / \mathrm{mL}$ is commonly considered a diagnostic threshold for measurement organisms in urine [33]. Principal component analysis (PCA) was carried out to visualize the spectral differences [34].Combined with PCA statistical analysis, SERS spectra of 10 random spots from E.coli exposed and unexposed at $4 \mu \mathrm{g} / \mathrm{mL}$ of ampicillin, and 10 random spots from $\mathrm{Amp}^{-}$- E.coli and Amp ${ }^{+}$- E.coli exposed at $4 \mu \mathrm{g} / \mathrm{mL}$ of ampicillin were used for antibiotic susceptibility testing.

\subsection{Instrument}

The "as-prepared" AuNR and Au@AgNR were diluted for longitudinal surface plasmon resonance (LSPR) detection, which were collected with a Shimadzu UV2600 UV-vis-NIR spectrometer. In addition, the UV-vis-NIR spectrometer was also used to measure OD of E.coli suspension. Transmission electron microscopy (TEM) images were 


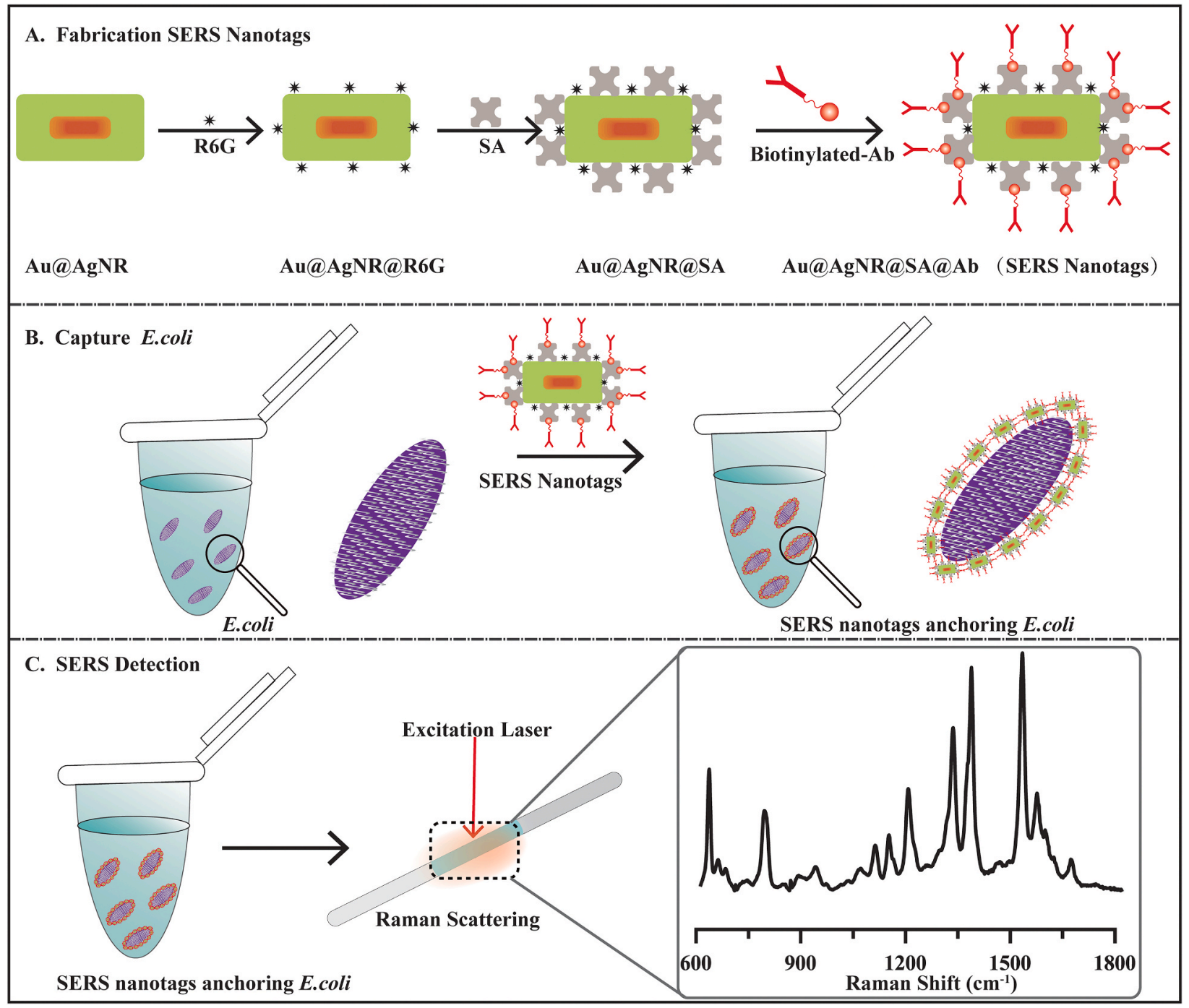

Fig. 1. Diagram of the preparation process of functionalized Au@AgNR as SERS nanotag and their attachment to E.coli.

determined by JEM-1011 TEM operating at $100 \mathrm{kV}$. The TEM samples at an appropriate concentration, including AuNR, Au@AgNR and Au@AgNR anchored E.coli, were dropped onto the copper grids. The zeta potential measurements were performed using a Brookhaven Zeta PALS instrument. Fluorescence microscope was used for monitoring the attachment of the SERS nanotags onto E. coli. The fluorescence detection filter sets were configured to an excitation wavelength of 555-580 nm (filter set 20HE, CarlZeiss). Axio Cam MRm was used to record fluorescence videos at an exposure time of $0.7 \mathrm{~s}$ with a multi-dimensional acquisition mode in the Axio-vision 4.7.1 release software (Carl Zeiss MicroImaging, Inc.). SERS measurements were performed via DXR Raman Microscope (Thermo Scientific) with laser wavelength at 785 $\mathrm{nm}$. The power of laser was set at $20 \mathrm{~mW}$, and the accumulation time was kept at $5 \mathrm{~s}$ for all experiments.

\section{Results and discussion}

\subsection{Fabrication of Au@Ag core-shell nanorods (Au@AgNR)}

AuNR are particularly attractive due to the unique optical properties and facile growth methods, which have been extended to many fields, such as biomedical sensor, photothermal therapy and so on $[35,36]$. Compared with Au nanocrystals, Ag nanocrystals can support localized plasmon resonances in the near-ultraviolet spectral range and exhibit much stronger electric field enhancements [7]. These features make $\mathrm{Ag}$ nanocrystals possess better performance in enhancing Raman scattering application, fluorescence application and so on [37]. However, the uniformity and stability of $\mathrm{Ag}$ nanocrystals are not as good as those of $\mathrm{Au}$ nanocrystals [38]. Herein, the anisotropic Au@AgNR are expected to improve the uniformity and stability of Ag nanocrystals, in which $\mathrm{Au}$ nanorods with great chemical stability and tunable longitudinal plasmon wavelengths can function as excellent supports for the formation of Ag shells.

Fig. 2A showed time dependent spectral changes. At $120 \mathrm{~min}$, after the addition of AuNR colloidal solution, the spectral changes have stopped. The Au@AgNR contributed separation of four extinction band subsequently at around 587, 489, 410 and $345 \mathrm{~nm}$. We define the plasmon bands sequentially as peaks $1,2,3,4$, respectively. The longitudinal and transverse plasmon resonance wavelengths of AuNR were around 786 and $512 \mathrm{~nm}$, respectively. Upon silver coating, the peak 1 has been assigned to the longitudinal dipolar plasmon mode, which blue shift from around around $786 \mathrm{~nm}$ to around $587 \mathrm{~nm}$. The peak 2 can be assigned to transverse dipolar modes, which blue shift from around 512 $\mathrm{nm}$ to around $489 \mathrm{~nm}$. The peaks 3 and 4 can both be ascribed to an octupolar mode [7], which remain nearly unchanged at around 410 and $345 \mathrm{~nm}$. In addition, an obvious color change occurred during silver coating, where the color of solution changes from pink to brown (Fig. S1). Fig. 2B and Fig. 2C clearly indicated the process of silver 
A

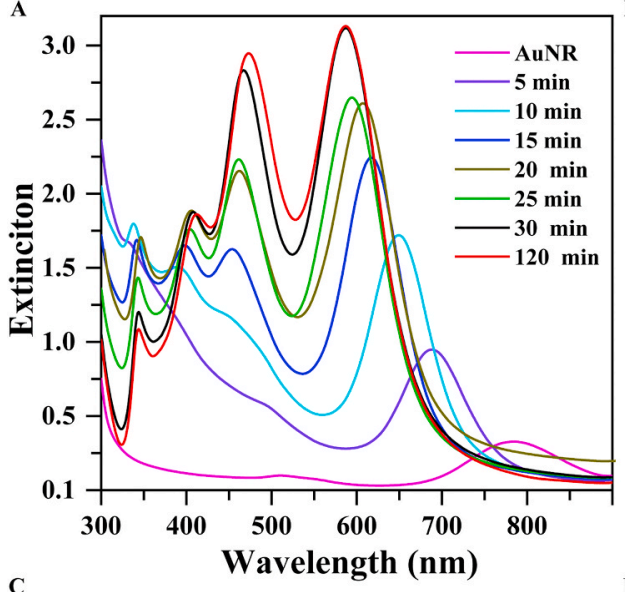

C

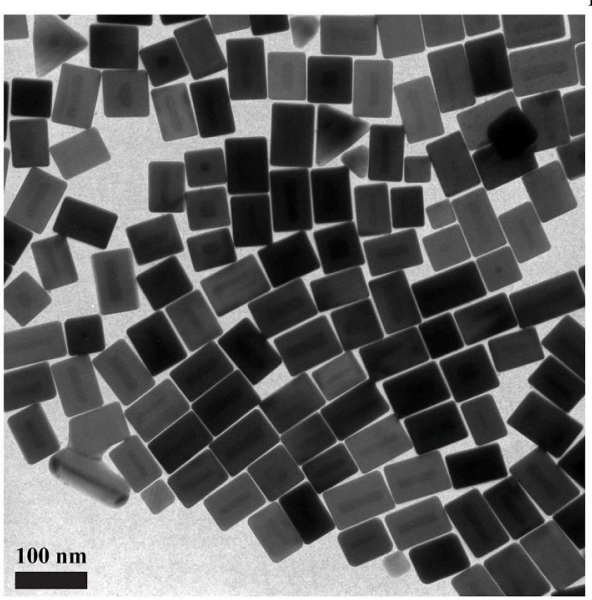

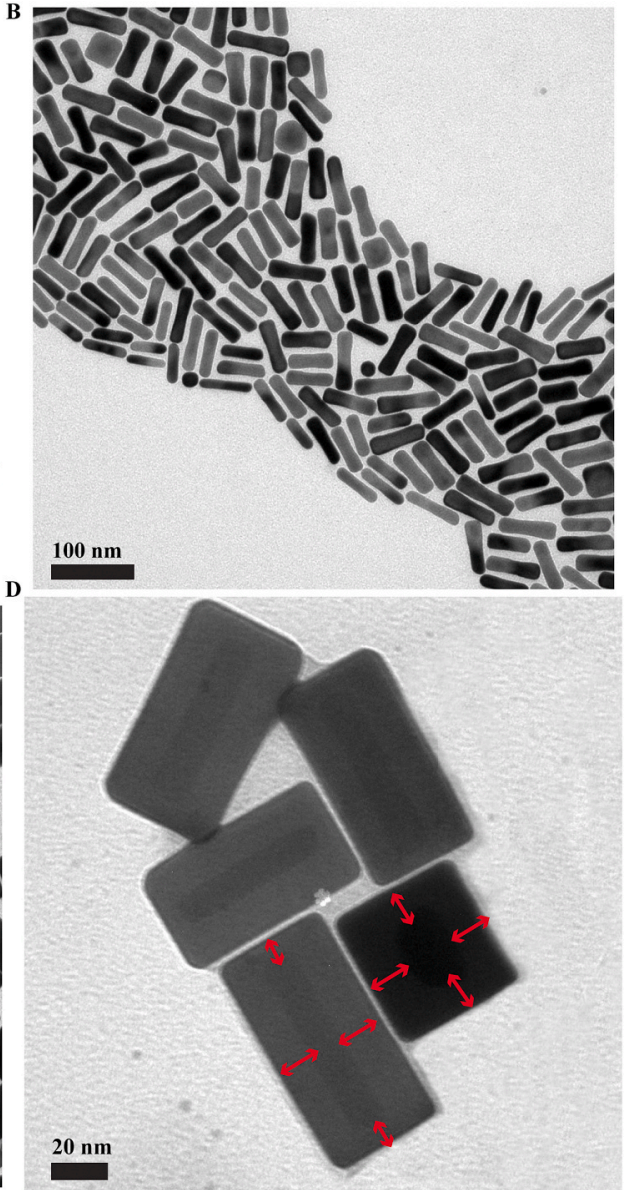

Fig. 2. (A) Extinction spectra of AuNR and Au@AgNR obtained by using $0.01 \mathrm{M} \mathrm{AgNO}_{3}$ solution at different time (5 min, $10 \mathrm{~min}, 15 \mathrm{~min}, 20 \mathrm{~min}, 25 \mathrm{~min}, 30 \mathrm{~min}$, 120 min), (B),(C) TEM images of AuNR and Au@AgNR, respectively, and (D) the enlarged TEM image of Au@AgNR.

coating on the AuNR. As seed nanoparticles, AuNR showed good uniformity, with average length of $69 \pm 6 \mathrm{~nm}$, average diameter of $17 \pm 2$ $\mathrm{nm}$, and aspect ratio of $4.1 \pm 1$. The Au@AgNRs was modeled as cuboidal shape, with slightly rounded corners and edges, which had narrow shape and size distributions. There were also some Au@AgNRs oriented vertically on the TEM grids. Their average length, diameter, and aspect ratio determined from the TEM images were $76 \pm 8 \mathrm{~nm}, 44 \pm$ $3 \mathrm{~nm}$, and 2, respectively (Table S1). As shown in Fig. 2D, the average shell thicknesses were 7.5 and $5.0 \mathrm{~nm}$ at the side and ends, respectively.

\subsection{Design of Au@AgNR SERS nanotags platform}

Fig. 1 provided the procedure for preparing SERS nanotag systems based on sandwich nanohybrids stepwise, which can bind to target bacteria. In brief, the Au@AgNRs mentioned above was functionalized with Raman reporter molecule, R6G and streptavidin in sequence, forming streptavidin-functionalized Au@AgNRs (Au@AgNR@SA). The biotinylated antibodies can be specially linked to the Au@AgNR@SA via strong noncovalent interaction [39], forming SERS nanotags (Au@AgNR@SA@Ab). In each experiment, bacteria sample was incubated with the SERS nanotag, allowing the SERS nanotags anchor onto the bacteria. The conjugation process at each stage was obtained using extinction spectroscopy, and zeta potential analysis. In Fig. 3A, peak 1 blue shifted from around 587 to $551 \mathrm{~nm}$, along with peak broadening. The peak 2 blue shifted from around 489 to $470 \mathrm{~nm}$. As shown in Fig. 3B, the zeta potentials of Au@AgNRs were observed at each stage. When LA was modified on the Au@AgNRs surface, the zeta potential of the functionalized Au@AgNRs complex decreased from +64 to +45 . The isoelectric point (pI) of streptavidin is $\mathrm{pH}$ 6.0, which is lower than the $\mathrm{pH}$ value of the complex. Therefore, after the modification of streptavidin onto the Au@AgNRs@LA surface, the zeta potential of the complex decreased to +35 . The changes in zeta potential confirmed the successful attachment of biotinylated antibody to the Au@AgNRs. With the help of biotinylated antibody, the SERS nanotags was capable of anchoring onto E.coli efficiently. Fig. 1 illustrated the typical Raman spectrum of such analytical platform, where Raman bands at around $612,774,1362,1517$, and $1651 \mathrm{~cm}^{-1}$ were from the quintessential vibrational modes of R6G [27]. R6G is chosen not only as SERS reporter but also as fluorescence dye molecule. Fig. 3C showed confocal fluorescence microscopy images of the $E$. coli bound by SERS nanotags (Fig. S2). As presented in Fig. 3D, the enlarged TEM image of single E. coli can further confirm that there were hundreds of SERS nanotags anchoring onto each $E$. coli on average, which can create sufficient hot spots to further amplify Raman signals for rapid detection of $E$. coli [40]. In comparison, Au@AgNRs@R6G can capture E. coli through electrostatic interaction between the positively charged Au@AgNR and negatively charged $E$. coli. The SERS signal from $E$. coli attached by SERS nanotag is much stronger than that from $E$. coli attached by Au@AgNR@R6G. The reason is that very few Au@AgNRs@R6G nonspecifically absorbed on E. coli surface (Fig. S3).

\subsection{Ability of the functionalized $A u @ A g N R$ SERS nanotags to capture and detect bacteria}

Fig. 4A showed the corresponding Raman spectra for SERS nanotags targeted E. coli at different concentrations ranging from $\sim 10^{2}$ to $10^{7}$ CFU mL ${ }^{-1}$. Fig. 4B summarized the SERS intensity of $E$. coli complex at different concentration, which was measured with band intensity at 

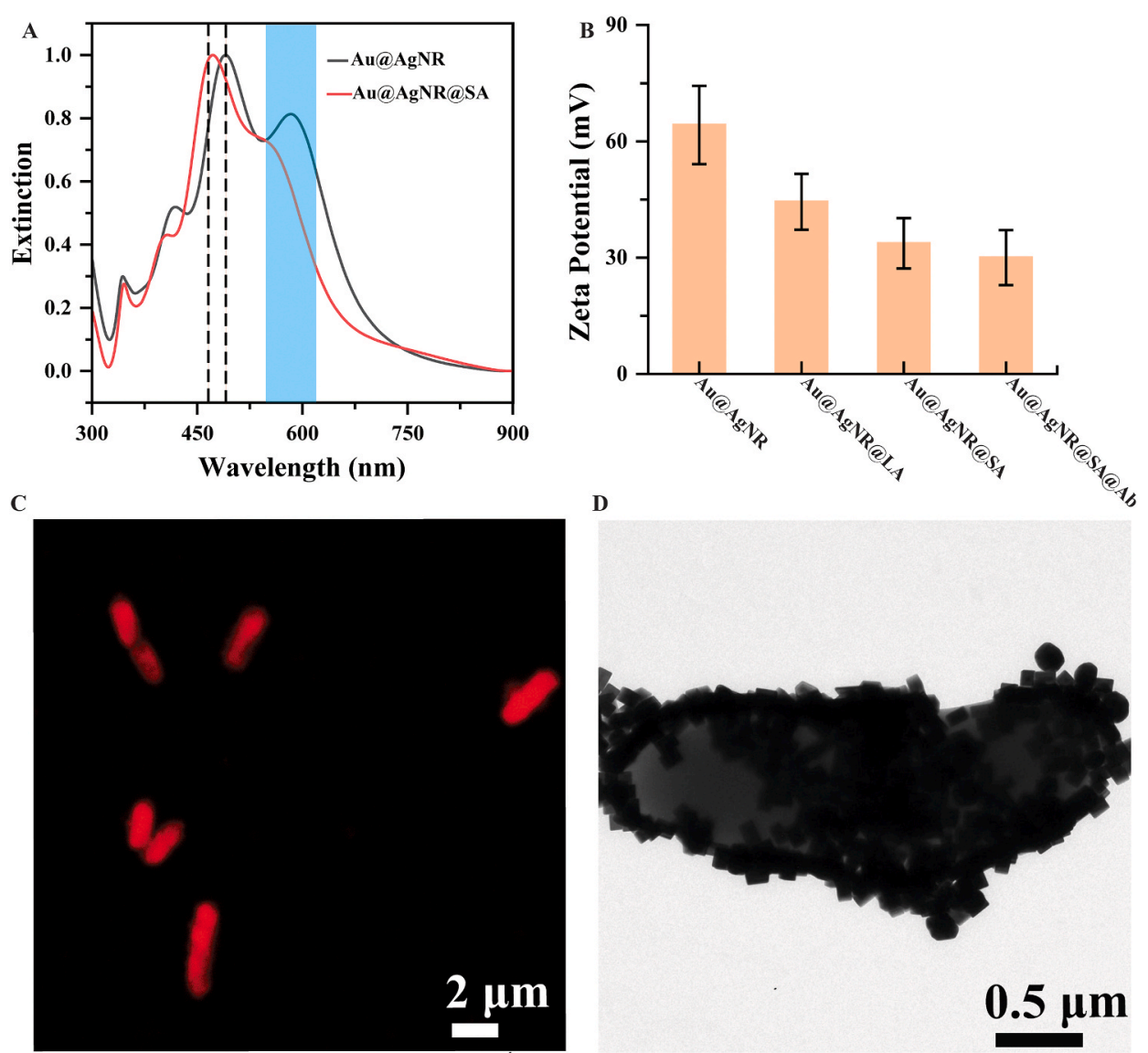

Fig. 3. (A) UV-vis spectra of Au@AgNR and Au@AgNR@SA. (B), The statistical results of the zeta potential for functionalization process of SERS nanotag. (C) Confocal fluorescence image of $E$. coli anchored by SERS nanotag. (D) TEM image of single $E$. coli anchored by SERS nanotag.

$\sim 1517 \mathrm{~cm}^{-1}$. In Fig. 4C, it was noticeable that a good linearity between the logarithm of bacteria concentration and SERS intensity of E. coli complex, as follows: $\mathrm{RI}=3865+193 \log C\left(\mathrm{R}^{2}=0.97\right)$, where RI (Raman intensity, RI) stands for the Raman intensity collected from the E.coli complex at different concentration. The $\log \mathrm{C}$ stands for logarithm of E. coli concentration. Herein, RI was proportional to $\log \mathrm{C}$, rather than directly proportional to bacteria concentration. It was worthwhile to point out that Raman intensity at $1517 \mathrm{~cm}^{-1}$ from Au@AgNR@R6G was less than 2400, which was much weaker than that from E.coli complex (Fig. S3). As a proof-of-concept to demonstrate the potential application of the SERS nanotag platform in biological environment, C57BL/6 mouse blood samples with SERS nanotags were spiked with E.coli at $10^{4}$ CFU $\mathrm{mL}^{-1}$ for testing. Fig. 4D exhibited some red blood cells with a diameter of 7-8 $\mu \mathrm{m}$, whereas no bacteria could be identified. The SERS spectra were recorded in Fig. 4E, where the prominent band of SERS nanotags bound $E$. coli can be clearly seen. The result was consistent with those of bacteria collected from PBS. Meanwhile, the SERS signal of E.coli with Au@AgNRs@R6G is much weaker than that from E.coli anchored by SERS nanotag. No characteristic vibrational bands were obtained at the same bacterial concentration mixed with water. The interference and nonspecific signal of the matrices were negligible. It was also done to determine whether E.coli detection is affected by a difference between the diluent used to prepare the standard curve (PBS) and the real sample matrix (tap water). This experiment was performed by spiking of PBS and tap water with $10^{2}, 10^{4}$ and $10^{6} \mathrm{CFU} / \mathrm{mL}$ of E.coli, and then the spiked samples were evaluated under the same condition in single experiment. As shown in Fig. S4, the obtained results for target analyte in tap water was similar to that in PBS. Furthermore, the ability of SERS nanotags to capture the $E$. coli was investigated. Following the method previously reported, capture efficiency was calculated based on the difference of colony number in E.coli supernatant of test solution before and after attachment. The capture efficiency of SERS nanotags and - Au@AgNRs@R6G were achieving 71.03\% and 56.41\%, respectively (Fig. S5).According to previous reports, the label-free bacteria detection based on polyethylenimine (PEI)-modified Au-coated magnetic microspheres $\left(\mathrm{Fe}_{3} \mathrm{O}_{4} @ \mathrm{Au} @ \mathrm{PEI}\right)$ showed high capture efficiency as much as $66 \%$ [41]. Herein the SERS nanotags offered much better capture efficiency. In practical application, researchers focus on the reproducibility and specificity of the SERS nanotags. The excellent reproducibility is demonstrated by slight variation of SERS nanotags from different batches (Fig. S6). The specificity of the SERS nanotag system verus another bacteria such as Proteus vulgaris and Staphylococcus aureus at $10^{4} \mathrm{CFU} / \mathrm{mL}$ were demonstrated (Fig. S7). The intensity SERS signal at $1517 \mathrm{~cm}^{-1}$ of E.coli was much stronger than that of Proteus vulgaris (P.vulgaris) and Staphylococcus aureus (S. aureus). The finding confirmed that the specificity of the SERS nanotag system toward E.coli, which resulted from biotinylated antibody specific recognition toward E.coli.

\subsection{Rapid antibiotic susceptibility testing (AST) of bacteria}

While bacteria are exposed to antibiotic, metabolic processes are perturbed, vital bacterial functions are inhibited, and bacterial growth is stopped [42]. Two experimental samples were adopted, which were $\mathrm{Amp}^{-}$-E. coli and Amp ${ }^{+}$-E. coli, respectively. The MIC of ampicillin for the $\mathrm{Amp}^{-}-E$. coli is $4 \mu \mathrm{g} / \mathrm{mL}$ and the MIC for $\mathrm{Amp}^{+}-E$. coli is $>4 \mu \mathrm{g} / \mathrm{mL}$, which were recommended in previous reports $[9,43]$. The SERS intensity was recorded during incubation time, in which Raman band at $1517 \mathrm{~cm}^{-1}$ has been selected as marker band. Fig. 5A showed the growth curve from SERS plots of Amp ${ }^{-}$-E. coli complex exposed (red line) and 
unexposed (black line) at $4 \mu \mathrm{g} / \mathrm{mL}$ of ampicillin. There were systematic changes occurred in the SERS intensity as response to the antibiotics. It is at $3.0 \mathrm{~h}$ when there is notable difference between the exposed and unexposed conditions. Fig. S8 displayed SERS spectra for Amp ${ }^{-}-E$. coli exposed and unexposed at $4 \mu \mathrm{g} / \mathrm{mL}$ of ampicillin at $3.0 \mathrm{~h}$. PCA can transform a set of observations into a set of values of variables called principal components, where first principal component explains the highest amount of variance in the data [12]. In our case, the data set referred to band intensity measured at around 612, 774, 1362, 1517, and $1651 \mathrm{~cm}^{-1}$. Antibiotic effect can be confirmed in Fig. 5B, where the PCA of Raman intensity can clearly exhibit the difference between spectra of $A^{-} p^{-}-E$. coli incubated with and without $4 \mu \mathrm{g} / \mathrm{mL}$ of ampicillin at $3.0 \mathrm{~h}$. The antibiotic was reflected by the positive shift and negative shift of spectra in PC1, indicating good separation between exposed and unexposed condition [44]. In the work, it only took $3.0 \mathrm{~h}$ to observe the variation between growing and inhibited bacteria, when the bacteria were in exponential growth phase. Fig. $5 \mathrm{C}$ displayed the growth curve from SERS plots for Amp ${ }^{-}-E$. coli complex (red line) and $\mathrm{Amp}^{+}-E$. coli complex (black line) at $4 \mu \mathrm{g} / \mathrm{mL}$ of ampicillin. It should be noted that the growth curve for $\mathrm{Amp}^{+}-E$. coli were identical to
$\mathrm{Amp}^{-}$-E. coli unexposed to some extent. Whereas the growth process for $\mathrm{Amp}^{-}-$E. coli treated with $4 \mu \mathrm{g} / \mathrm{mL}$ of ampicillin was inhibited. It is at $3.5 \mathrm{~h}$, when we can observe the variation between growing and inhibited bacteria. At this time point, SERS spectra obtained from in Fig. S8. As illustrated in Fig. 5D, Raman intensity of $\mathrm{Amp}^{-}$-E.coli complex and $\mathrm{Amp}^{+}$-E.coli complex were used in the PCA algorithm. The loading profiles of PC1-PC2 indicated that the potential for distinguishing $\mathrm{Amp}^{-}$-E.coli from $\mathrm{Amp}^{+}$-E. coli. SERS nanotag coupled with the multivariate statistics displayed excellent performace, indicating that our proposed platform has great potential for providing sensitive antibiotic susceptibility testing. The established phenotypical AST standard methods involve broth microdilution assays, gradient diffusion and disc diffusion, which takes at least one day to readout. Nowadays, commercial automated systems such as MicroScan WalkAway will take 6-16 $\mathrm{h}$ to shorten the wait time in clinical application [45]. In our work, the changes of Raman intensity can reflect inhibition and growth of the bacteria directly, which cost less than $3.5 \mathrm{~h}$ to discriminate antibiotic resistance. Compared with previous reported methods, our SERS nanotag platform enables faster AST analysis, which promises to provide early initialization of effective antibiotic therapy.
A

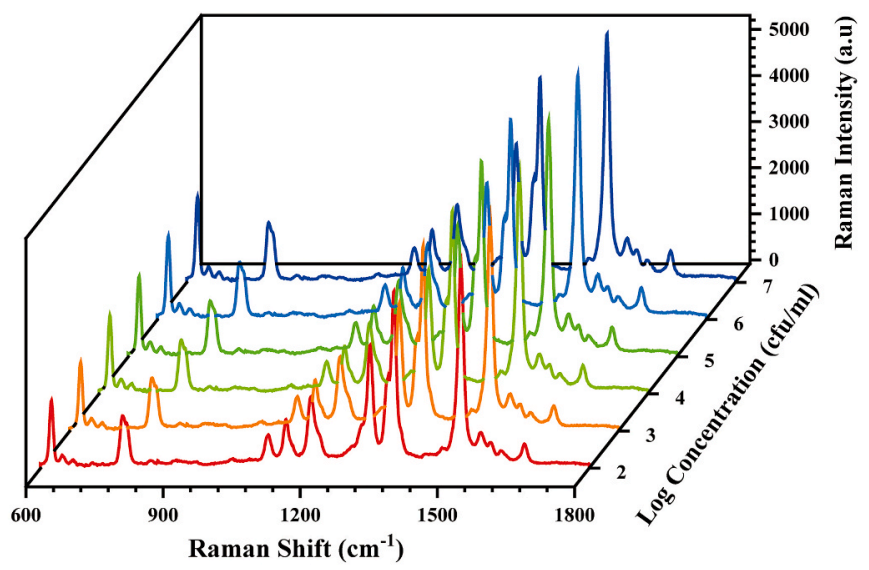

\section{B}

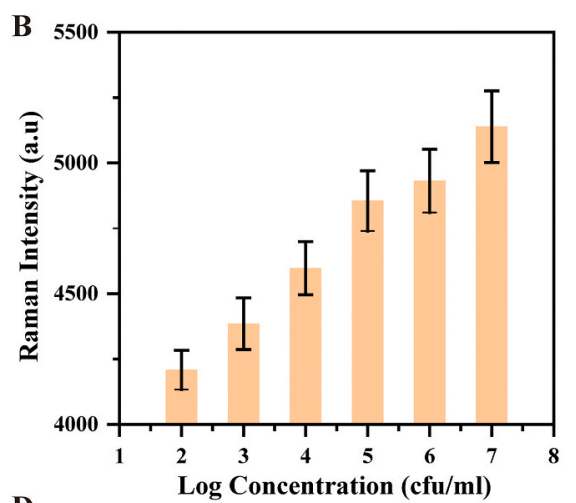

D

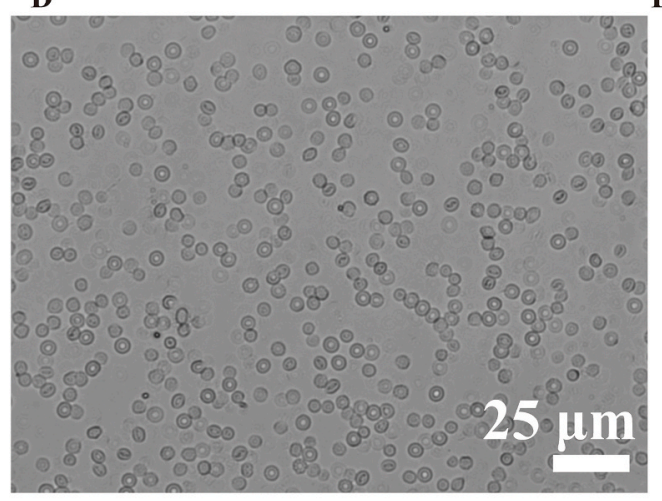

C
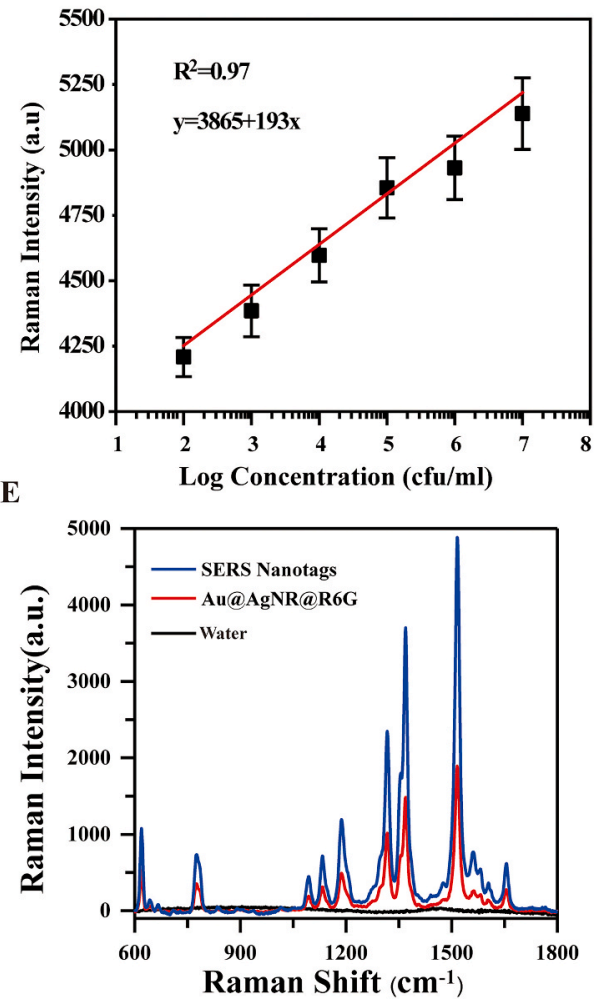

Fig. 4. (A) The SERS spectra of E.coli complex at different concentration ranging from $10^{7}$ to $10^{2}$ $\mathrm{CFU} / \mathrm{mL}$. (B) The intensity of SERS signal at $1517 \mathrm{~cm}^{-1}$ of E.coli complex mentioned in Fig. 4A. (C) Corresponding linear fitting of Raman intensity with logarithmic E.coli concentration. (D) Optical microscopy image of SERS nanotag incubated with mouse blood containing E.coli at $10^{4} \mathrm{CFU} / \mathrm{mL}$. (E) The SERS spectra of mouse blood containing E.coli at $10^{4} \mathrm{CFU} / \mathrm{mL}$ spiked with SERS nanotag, Au@AgNRs@R6G and water. 

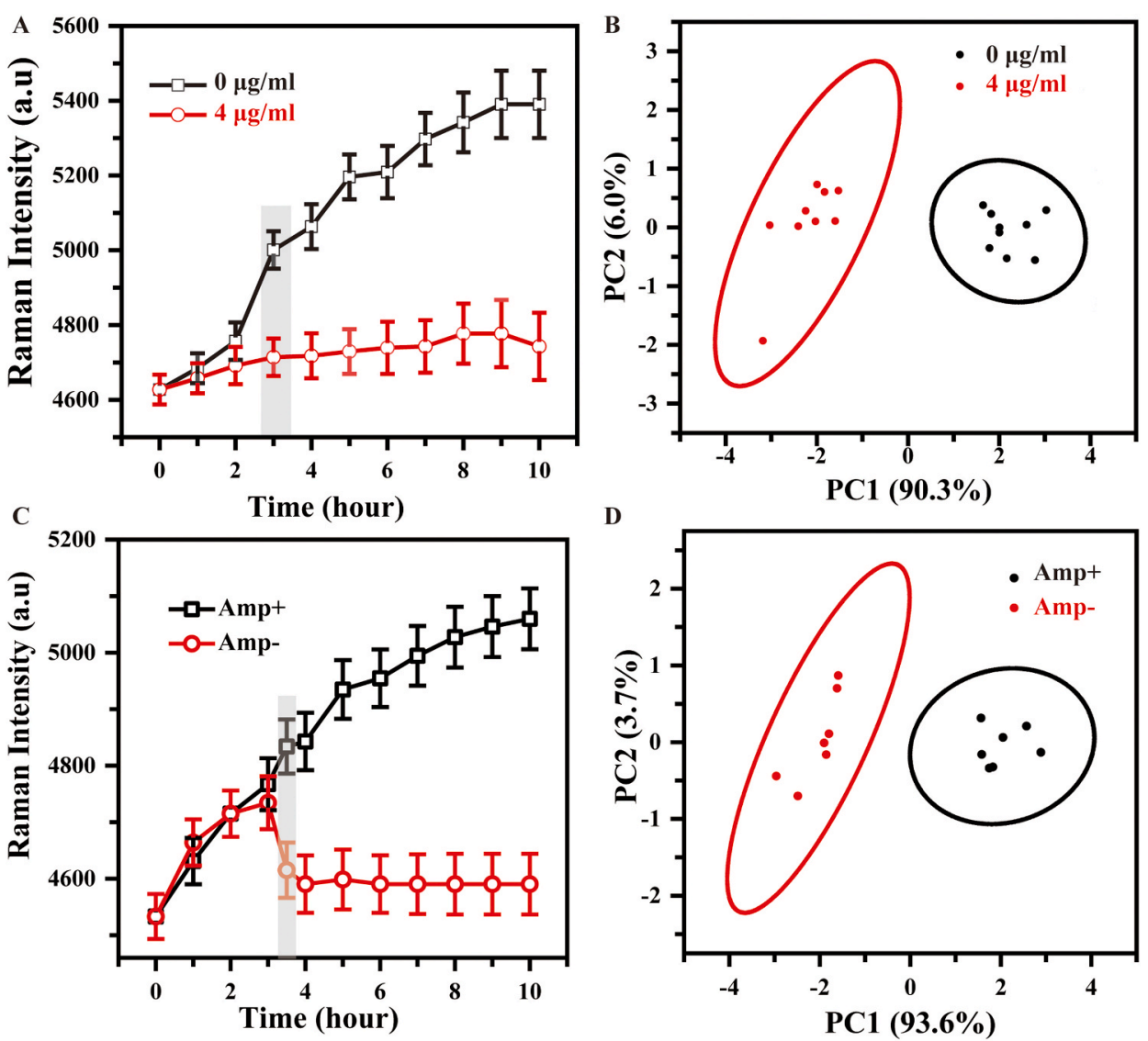

Fig. 5. (A) The growth curve from SERS plots for $\mathrm{Amp}^{-}-$E. coli complex exposed (red line) and unexposed (black line) at $4 \mu \mathrm{g} / \mathrm{mL}$ of ampicillin. (B) Combinations of PCA loading profiles of $\mathrm{Amp}^{-}-$E. coli complex exposed (red line) and unexposed (black line) at $4 \mu \mathrm{g} / \mathrm{mL}$ of ampicillin at $3 \mathrm{~h}$. (C) The growth curve from SERS plots for $\mathrm{Amp}^{+}-E$. coli complex (black line) and Amp ${ }^{-}$. E. coli complex (red line) exposed at $4 \mu \mathrm{g} / \mathrm{mL}$ of ampicillin. (D) Combinations of PCA loading profiles of $\mathrm{Amp}^{+}-E$. coli complex (black line) and $\mathrm{Amp}^{-}-E$. coli complex exposed at $4 \mu \mathrm{g} / \mathrm{mL}$ of ampicillin at $3.5 \mathrm{~h}$. (For interpretation of the references to color in this figure legend, the reader is referred to the Web version of this article.)

\section{Conclusions}

In summary, we developed a SERS-active Au@AgNR tag platform for ultrasensitive bacteria detection and antibiotic-susceptibility testing. By aid of biotinylated antibodies, the SERS platform could obtain closest attachment on E.coli as much as possible, forming high-accessibly hot junctions from the nanogaps. We also demonstrated that the SERS nanotag platform possess good reproducibility, specificity and bacterial capture efficiency, arriving at $71.03 \%$. The SERS nanotags was successfully applied to detect E.coli with limit of detection as low as $10^{2}$ $\mathrm{CFU} / \mathrm{mL}$. Furthermore, coupled with PCA chemometric analysis, the strategy was successfully used to discriminate antibiotic resistance within 3.5 h. For practical applications, E.coli was spiked into blood, which can be readily distinguished. Herein, E. coli are selected as the model bacteria, indicated that our SERS nanotag platform can be used for the other kind of bacteria detection and antibiotic susceptibility testing, which open an avenue for promising to provide early initialization of effective antibiotic therapy.

\section{CRediT authorship contribution statement}

Liyan Bi: Conceptualization, Validation, Formal analysis, Investigation, Writing - original draft, Funding acquisition. Xiao Wang: Data curation, Methodology, Investigation, Software. Xiaowei Cao: Conceptualization, Software, Visualization. Luying Liu: Validation, Formal analysis, Investigation, Validation. Congcong Bai: Formal analysis, Investigation. Qingyin Zheng: Conceptualization, Validation. Jaebum Choo: Conceptualization, Funding acquisition, Methodology. Lingxin Chen: Conceptualization, Funding acquisition, Methodology, Writing - review \& editing.

\section{Declaration of competing interest}

The authors declare no competing financial interest.

\section{Acknowledgments}

We gratefully acknowledge support from the National Natural Science Foundation of China (Grant No. 21605005, 81530030, 81873697, 21976209), National Research Foundation of Korea (Grant No. 2019R1A2C3004375 and 2018M3A7B4071203), Research Initiation Fund of Binzhou Medical University (Grant No. BY2019KYQD39) and Taishan Scholar Project Special Funding (Grant No. ts20190962).

\section{Appendix A. Supplementary data}

Supplementary data to this article can be found online at https://doi. org/10.1016/j.talanta.2020.121397.

\section{References}

[1] A.G. Bozkurt, G.G. Buyukgoz, M. Soforoglu, U. Tamer, Z. Suludere, I.H. Boyaci, Alkaline phosphatase labeled SERS active sandwich immunoassay for detection of Escherichia coli, Spectrochim. Acta Mol. Biomol. Spectrosc. 194 (2018) 8-13.

[2] Z. Lin, X. Zhao, J. Huang, W. Liu, Y. Zheng, X. Yang, et al., Rapid screening of colistin-resistant Escherichia coli, Acinetobacter baumannii and Pseudomonas aeruginosa by the use of Raman spectroscopy and hierarchical cluster analysis, Analyst 144 (2019) 2803-2810.

[3] A.J. Kaasch, G. Barlow, J.D. Edgeworth, V.G. Fowler, M. Hellmich, S. Hopkins, et al., Staphylococcus aureus bloodstream infection: a pooled analysis of five prospective, observational studies, J. Infect. 68 (3) (2014), 242-251.

[4] R.M. Jarvis, R. Goodacre, Characterisation and identification of bacteria using SERS, Chem. Soc. Rev. 37 (2008) 931-936.

[5] H.N. Abdelhamid, H.-F. Wu, Multifunctional graphene magnetic nanosheet decorated with chitosan for highly sensitive detection of pathogenic bacteria, J. Mater. Chem. B 1 (2013) 3950-3961. 
[6] F.R. Madiyar, S. Bhana, L.Z. Swisher, C.T. Culbertson, X. Huang, J. Li, Integration of a nanostructured dielectrophoretic device and a surface-enhanced Raman probe for highly sensitive rapid bacteria detection, Nanoscale 7 (2015) 3726-3736.

[7] R. Jiang, H. Chen, L. Shao, Q. Li, J. Wang, Unraveling the evolution and nature of the plasmons in (Au core)-(Ag shell) nanorods, Adv. Mater. 24 (2012) OP200-OP207.

[8] D. Cheng, M. Yu, F. Fu, W. Han, G. Li, J. Xie, et al., Dual recognition strategy for specific and sensitive detection of bacteria using aptamer-coated magnetic beads and antibiotic-capped gold nanoclusters, Anal. Chem. 88 (2016) 820-825.

[9] R.T. Hayden, L.K. Clinton, C. Hewitt, T. Koyamatsu, Y. Sun, G. Jamison, et al., Rapid antimicrobial susceptibility testing using forward laser light scatter technology, J. Clin. Microbiol. 54 (2016) 2701-2706.

[10] S. Qiu, Z. Lin, Y. Zhou, D. Wang, L. Yuan, Y. Wei, et al., Highly selective colorimetric bacteria sensing based on protein-capped nanoparticles, Analyst 140 (2015) 1149-1154.

[11] U.C. Schroder, C. Beleites, C. Assmann, U. Glaser, U. Hubner, W. Pfister, et al., Detection of vancomycin resistances in enterococci within $31 / 2$ hours, Sci. Rep. 5 (2015) 1-7.

[12] L. Qiu, W. Wang, A. Zhang, N. Zhang, T. Lemma, H. Ge, et al., Core-shell nanorod columnar array combined with gold nanoplate-nanosphere assemblies enable powerful in situ SERS detection of bacteria, ACS Appl. Mater. Interfaces 8 (2016) 24394-24403.

[13] S. Stöckel, J. Kirchhoff, U. Neugebauer, P. Rösch, J. Popp, The application of Raman spectroscopy for the detection and identification of microorganisms, J. Raman Spectrosc. 47 (2016) 89-109.

[14] A. Walter, A. Marz, W. Schumacher, P. Rosch, J. Popp, Towards a fast, high specific and reliable discrimination of bacteria on strain level by means of SERS in a microfluidic device, Lab Chip 11 (2011) 1013-1021.

[15] P.X. Wang, S. Pang, J.H. Chen, L. McLandsborough, S.R. Nugen, M.T. Fan, et al., Label-free mapping of single bacterial cells using surface-enhanced Raman spectroscopy, Analyst 141 (2016) 1356-1362.

[16] B. Guven, N. Basaran-Akgul, E. Temur, U. Tamer, I.H. Boyaci, SERS-based sandwich immunoassay using antibody coated magnetic nanoparticles for Escherichia coli enumeration, Analyst 136 (2011) 740-748.

[17] S.A. Khan, A.K. Singh, D. Senapati, Z. Fan, P.C. Ray, Targeted highly sensitive detection of multi-drug resistant salmonella DT104 using gold nanoparticles, Chem. Commun. 47 (2011) 9444-9446.

[18] C. Wang, J. Wang, M. Li, X. Qu, K. Zhang, Z. Rong, et al., A rapid SERS method for label-free bacteria detection using polyethylenimine-modified Au-coated magnetic microspheres and Au@Ag nanoparticles, Analyst 141 (2016) 6226-6238.

[19] D. Lin, T. Qin, Y. Wang, X. Sun, L. Chen, Graphene oxide wrapped SERS tags: multifunctional platforms toward optical labeling, photothermal ablation of bacteria, and the monitoring of killing effect, ACS Appl. Mater. Interfaces 6 (2014) 1320-1329.

[20] C. Catala, B. Mir-Simon, X. Feng, C. Cardozo, N. Pazos-Perez, E. Pazos, et al., Online SERS quantification of Staphylococcus aureus and the application to diagnostics in human fluids, Adv. Mater. Technol. 1 (2016) 1600163.

[21] H. Kearns, R. Goodacre, L.E. Jamieson, D. Graham, K. Faulds, SERS detection of multiple antimicrobial-resistant pathogens using nanosensors, Anal. Chem. 89 (2017) 12666-12673.

[22] H. Zhou, D. Yang, N.P. Ivleva, N.E. Mircescu, R. Niessner, C. Haisch, SERS detection of bacteria in water by in situ coating with $\mathrm{Ag}$ nanoparticles, Anal. Chem. 86 (2014) 1525-1533

[23] F.L. Li, F. Li, D. Luo, W.H. Lai, Y.H. Xiong, H.Y. Xu, Biotin-exposure-based immunomagnetic separation coupled with nucleic acid lateral flow biosensor for visibly detecting viable Listeria monocytogenes, Anal. Chim. Acta 1017 (2018) 48-56.

[24] L.Y. Bi, J. Dong, W. Xie, W.B. Lu, W. Tong, L. Tao, et al., Bimetallic gold-silver nanoplate array as a highly active SERS substrate for detection of streptavidin/ biotin assemblies, Anal. Chim. Acta 805 (2013) 95-100.

[25] N. Karami, A. Martner, V.I. Enne, S. Swerkersson, I. Adlerberth, A.E. Wold, Transfer of an ampicillin resistance gene between two Escherichia coli strains in the bowel microbiota of an infant treated with antibiotics, J. Antimicrob. Chemother. 60 (2007) 1142-1145.

[26] X. Meng, H. Wang, N. Chen, P. Ding, H. Shi, X. Zhai, et al., A graphene-silver nanoparticle-silicon sandwich SERS chip for quantitative detection of molecules and capture, discrimination, and inactivation of bacteria, Anal. Chem. 90 (2018) 5646-5653.

[27] L.Y. Bi, Y.Q. Wang, Y. Yang, Y.L. Li, S.S. Mo, Q.Y. Zheng, et al., Highly sensitive and reproducible SERS sensor for biological pH detection based on a uniform gold nanorod array platform, ACS Appl. Mater. Interfaces 10 (2018) 15381-15387.

[28] C. Hamon, M. Postic, E. Mazari, T. Bizien, C. Dupuis, P. Even-Hernandez, et al., Three-dimensional self-assembling of gold nanorods with controlled macroscopic shape and local smectic B order, ACS Nano 6 (2012) 4137-4146.

[29] E. Temur, I.H. Boyac1, U. Tamer, H. Unsal, N. Aydogan, A highly sensitive detection platform based on surface-enhanced Raman scattering for Escherichia coli enumeration, Anal. Bioanal. Chem. 397 (2010) 1595-1604.

[30] S.H. Choi, J.W. Lee, S.J. Sim, Enhanced performance of a surface plasmon resonance immunosensor for detecting Ab-GAD antibody based on the modified self-assembled monolayers, Biosens. Bioelectron. 21 (2005) 378-383.

[31] Y. Wang, B. Yan, L. Chen, SERS tags: novel optical nanoprobes for bioanalysis, Chem. Rev. 113 (2013) 1391-1428.

[32] M. Focsan, A. Campu, A.M. Craciun, M. Potara, C. Leordean, D. Maniu, et al., A simple and efficient design to improve the detection of biotin-streptavidin interaction with plasmonic nanobiosensors, Biosens. Bioelectron. 86 (2016) $728-735$.

[33] R.T. Hayden, L.K. Clinton, C. Hewitt, T. Koyamatsu, Y. Sun, G. Jamison, et al., Rapid antimicrobial susceptibility testing using forward laser light scatter technology, J. Clin. Microbiol. 54 (2016) 2701-2706.

[34] J. Kirchhoff, U. Glaser, J.A. Bohnert, M.W. Pletz, J. Popp, U. Neugebauer, Simple ciprofloxacin resistance test and determination of minimal inhibitory concentration within 2 h using Raman spectroscopy, Anal. Chem. 90 (2018) 1811-1818.

[35] X. Huang, S. Neretina, M.A. El-Sayed, Gold nanorods: from synthesis and properties to biological and biomedical applications, Adv. Mater. 21 (2009) 4880-4910.

[36] R. Bardhan, S. Lal, A. Joshi, N.J. Halas, Theranostic nanoshells: from probe design to imaging and treatment of cancer, Acc. Chem. Res. 44 (2011) 936-946.

[37] R.J. Stokes, A. Macaskill, P.J. Lundahl, W.E. Smith, K. Faulds, D. Graham, Quantitative enhanced Raman scattering of labeled DNA from gold and silver nanoparticles, Small 3 (2007) 1593-1601.

[38] C. Damm, D. Segets, G. Yang, B.F. Vieweg, E. Spiecker, W. Peukert, Shape transformation mechanism of silver nanorods in aqueous solution, Small 7 (2011) $147-156$.

[39] P.C. Weber, D.H. Ohlendorf, J.J. Wendoloski, F.R. Salemme, Structural origins of high-affinity biotin binding to streptavidin, Science 243 (1989) 85-88.

[40] H. Wang, Y. Zhou, X. Jiang, B. Sun, Y. Zhu, H. Wang, et al., Simultaneous capture, detection, and inactivation of bacteria as enabled by a surface-enhanced Raman scattering multifunctional chip, Angew Chem. Int. Ed. Engl. 54 (2015) 5132-5136.

[41] J. Wang, X. Wu, C. Wang, N. Shao, P. Dong, R. Xiao, et al., Magnetically assisted surface-enhanced Raman spectroscopy for the detection of Staphylococcus aureus based on aptamer recognition, ACS Appl. Mater. Interfaces 7 (2015) 20919-20929.

[42] A. Tannert, R. Grohs, J. Popp, U. Neugebauer, Phenotypic antibiotic susceptibility testing of pathogenic bacteria using photonic readout methods: recent achievements and impact, Appl. Microbiol. Biotechnol. 103 (2019) 549-566.

[43] J.V. Bugrysheva, C. Lascols, D. Sue, L.M. Weigel, Rapid antimicrobial susceptibility testing of Bacillus anthracis, Yersinia pestis, and burkholderia pseudomallei by use of laser light scattering technology, J. Clin. Microbiol. 54 (2016) 1462-1471.

[44] A. Novelli-Rousseau, I. Espagnon, D. Filiputti, O. Gal, A. Douet, F. Mallard, et al., Culture-free antibiotic-susceptibility determination from single-bacterium Raman spectra, Sci. Rep. 8 (2018) 3957.

[45] J. Kirchhoff, U. Glaser, J.A. Bohnert, M.W. Pletz, J. Popp, U. Neugebauer, Simple ciprofloxacin resistance test and determination of minimal inhibitory concentration within 2 h using Raman spectroscopy, Anal. Chem. 90 (2018) 1811-1818. 